\title{
Perceptual-motor coordination and adaptation during locomotion: Determinants of prism adaptation in hall exposure
}

\author{
GORDON M. REDDING \\ Illinois State University, Normal, Illinois \\ and \\ BENJAMIN WALLACE \\ Cleveland State University, Cleveland, Ohio
}

\begin{abstract}
Adaptation to prismatic displacement was examined under a number of conditions with locomotion in a hallway exposure. In general, total prism adaptation was inversely related to secondary cognitive load (presence or absence of mental arithmetic) and the relative magnitude of visual and proprioceptive shift depended upon the availability of visible sound sources in the hall environment. When the speaking experimenter was visible to the subject, visual shift was greater than proprioceptive shift, but when the experimenter was not visible and/or was silent, proprioceptive shift was greater than visual shift. No relationship has been detected between locomotion (walking rate) and either prism adaptation or cognitive load. These results are consistent with a model which assumes that the total adaptive response depends upon the available capacity to establish and maintain coordinative linkage between discordant systems, whereas locus of adaptive recalibration depends upon the direction of these coordinative linkages, discordance and adaptation occurring in the guided system(s). The available capacity for coordinative linkage depends upon cognitive load, whereas the directionality of linkage is independently determined by task structure. Locomotion is assumed to be mediated by a sensorimotor system that does not involve the distorted positional information.
\end{abstract}

The picture of perceptual-motor organization that has emerged from prism-adaptation research is both surprisingly simple and bewilderingly complex. The total adaptive response can usually be described in terms of local adaptive changes in a linear system (Howard, 1971, 1982) and additive contributions from the various components of such a serially organized system (e.g., Redding, 1978; Redding \& Wallace, 1976, 1978; Templeton, Howard, \& Wilkinson, 1974; Wallace, 1977; Wallace \& Redding, 1979; Welch, 1974; Welch, Choe, \& Heinrich, 1974; Wilkinson, 1971). However, the variety of local adaptation is almost indefinitely large, including change in visual proprioceptive, postural, locomotive, and auditory systems (for a review, see Welch, 1978). It is this variability in locus of adaptation that presents the greatest challenge to current theories of prism adaptation.

For example, a bewildering variety of exposure conditions have been shown to produce almost inexplicable reversals in the relative amounts of visual and proprioceptive adaptation to lateral displacement of the optic array.'

The research was supported in part by Grant 1-RO3-MH34383 from the National Institute for Mental Health to the second author. Special thanks are due Mary Persanyi for assistance in data collection. Reprint requests should be addressed to Gordon $M$. Redding, Department of Psychology, Illinois State University, Normal, IL 61761.
Hallway walking without sight of the body usually produces more visual than proprioceptive adaptation (e.g., Redding, Clark, \& Wallace, 1985; Redding \& Wallace, 1976), but stationary viewing of the feet produces exclusively visual adaptation (Craske, 1967; Craske \& Crawshaw, 1974, 1978). On the other hand, pointing at targets with the hand visible throughout the reaching movement tends to produce more proprioceptive than visual adaptation (e.g., Harris, 1965; Kalil \& Freedman, 1966), but pointing with a view of the hand only at the end of the reaching movement tends to produce more visual than proprioceptive adaptation (e.g., Uhlarik \& Canon, 1971).

Studies by Canon (1970) and Kelso, Cook, Olson, and Epstein (1975) provide a hint as to a possible reason for such variability. In the Canon study, subjects tracked the location of a visible sounding target with their right hand. In different conditions, subjects tracked either the optically displaced visual target or the pseudophonically displaced auditory target. Thus, in the different conditions, either the eye or the ear was the guiding system in the sense of specifying the target location for the tracking response and the other system was guided in the sense that it was under control of the guiding system. Situational variables determine whether a guided system emits an overt response, but even systems that are not overtly active may 
receive spatially coded information about the target. Discordance between such expected location and local sensory information about the achieved target prompts local recalibration of nonguiding systems. Note that the subject did not receive feedback about the accuracy of tracking (i.e., no sight of the right hand); there was no discordance and no basis for adaptation in this system. In the Canon study, adaptation was largely restricted to the nonguiding discordant (visual or auditory) system, and in a similar manipulation of the directionality of guidance between visual and proprioceptive (hand-head) systems, Kelso et al. found adaptation to be entirely restricted to the nonguiding discordant system.

The suggestion in these results is that when two discordant systems are linked to perform some coordination task, adaptive recalibration and discordance reduction tend to be localized in the perceptual mechanisms of the guided system, the direction of the guidance linkage being determined by task demands (cf. Hamilton, 1964; Howard \& Templeton, 1966, p. 380). The linearity of perceptualmotor organization and the dependence of locus of adaptation on the direction of guidance between coordinated systems suggests the following directionality-of-guidance model of prism adaptation (see also Redding et al., 1985; Redding \& Wallace, 1985).

\section{Directionality-of-Guidance Model}

The perceptual-motor system is assumed to be organized into a number of semi-autonomous sensorimotor systems, each operating within its own unique motor (M) space (see Figure 1). By "'motor space," we mean the local area of operation of a particular sensor and its immediately associated musculature. For example, the eyes and extraocular muscles may be considered to be one (eye-head) sensorimotor system whose motor space (i.e., the visual field) is defined by the range and metric of its sensors and effectors. Similarly, the collective function of sensors and effectors from hand to head, inclusively, may be considered another (hand-head) system whose operational area (motor space) has a different range and metric. Note carefully that it is the network of neural connections, not the particular sensors and effectors, which defines a sensorimotor system. For instance, hand-head and ear-head systems may share neck muscles, but the efferent-afferent connections with the auditory sensors define an ear-head motor space different from that of the hand-head system. Thus, each subsystem in the total perceptual-motor system is equipped with its own effectors and sensors and is capable of independent operation in restricted situations. For example, when we sit quietly reading, the visual (eye-head) system is largely indepen-

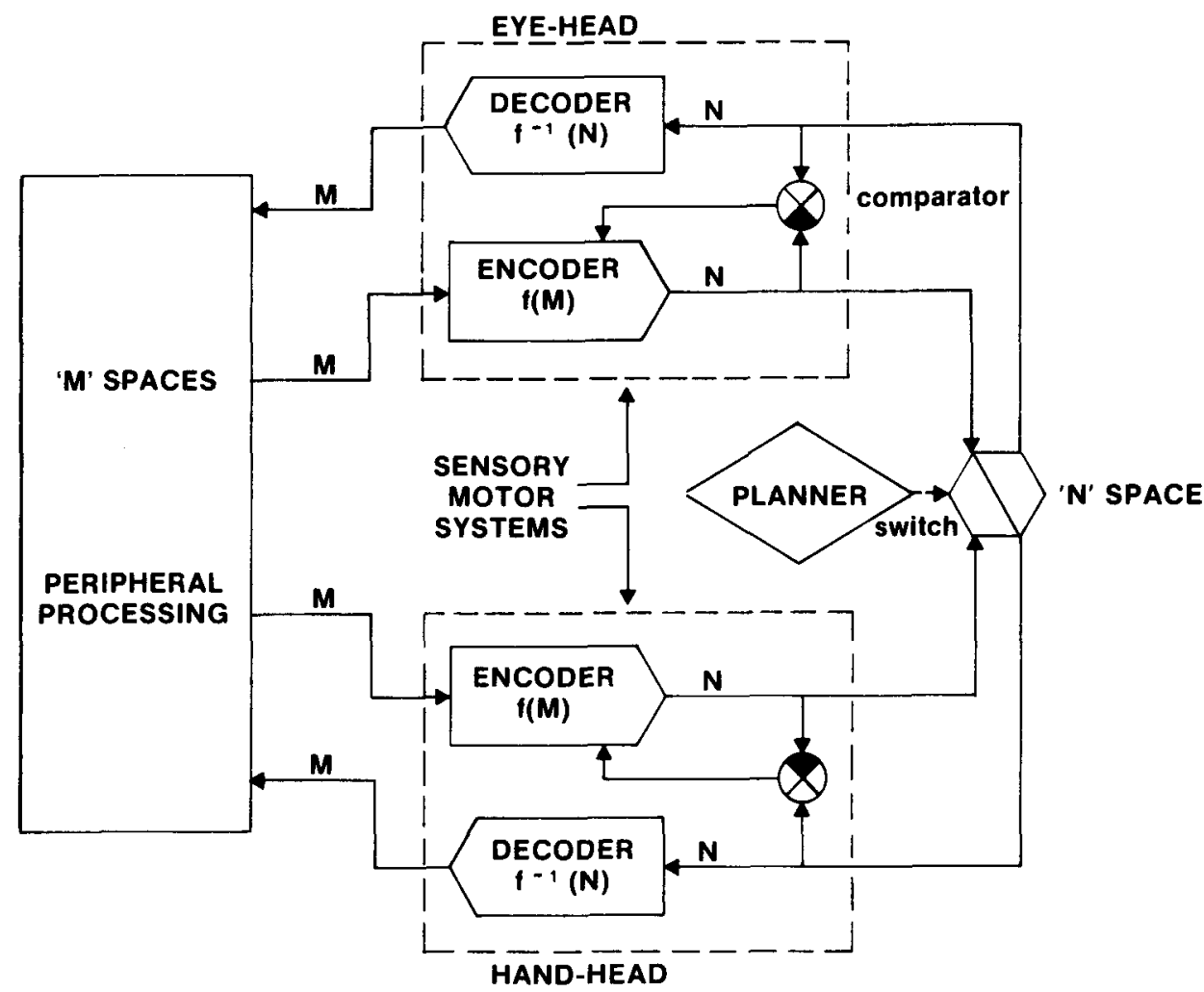

Figure 1. Directionality-of-guidance model. Coordination of sensorimotor systems during prism exposure produces spatial discordance in guided systems. Such discordance is removed by recalibration of local encoding operators that map unique motor $(M)$ spaces onto a noetic $(N)$ space common to the total perceptualmotor system. Coordinative linkage is shown set in the direction that would produce adaptation in the hand-head (proprioceptive) system. 
dent of any proprioceptive guidance. Conversely, when we adjust clothing without looking, proprioceptive systems (e.g., hand-head) are free of any visual guidance.

We further assume that cooperation among sensorimotor systems in situations of larger scope requires encoding and decoding operators that translate between the various metrically unique motor spaces via a single noetic (N) space, a metric common to all the various parts of the perceptual-motor system (Fisher, 1962a, 1962b; Howard, 1982). Noetic space permits central programming of perceptual-motor behavior with general commands that can be translated into coordinates of a specific sensorimotor system. For example, when we reach for a visual target, visual location may be first translated into noetic coordinates which are then sent to the hand, where they can be decoded into coordinates of the hand motor space. The reverse translation may be necessary when, for example, we look at our wristwatches.

Coordinative linkages between systems are assumed to be directional guidance linkages, represented in Figure 1 by the bidirectional "switch." That is, a given system cannot at the same time be guiding and guided by another system, although one system may guide several other systems in parallel (e.g., when we reach with both hands for the morning coffee). Linkage may be "automatic" for well-structured and highly habitual tasks, but variable or unusual tasks require limited-capacity central control processes (represented in Figure 1 by the "planner") to establish and maintain appropriate linkage between systems. $^{2}$

Now, when misalignments occur between systems, either because of natural dysmetria (e.g., Held \& Bossom, 1961; Robinson, 1976) or because of the artificial misalignment produced by prisms, spatial discordance between commanded and achieved positions in the guided system causes adaptive recalibration of the local encoding operator. As illustrated in Figure 1, the comparator in the guided hand-head system outputs a discrepancy between noetically coded efference and afference which prompts a gradual change in the local encoding operator such that the noetic coordinates of the hand are brought into alignment with the seen position of the hand (i.e., proprioceptive adaptation). In contrast, the guiding visual (eye-head) system receives no conflicting external commands, and there is no local discordance and no basis for adaptation in this system. Reversing the direction of the switch would produce visual adaptation (i.e., in the eyehead system).

\section{Cognitive Interference in Hall Exposure}

The directionality-of-guidance model assumes strategically flexible directionality of intersystem guidance, but a limited capacity to establish and maintain such intersystem linkage. In the prism exposure situation, normally unmonitored linkages between discordant systems produce errors. Reestablishment of accurate behavior requires feedback monitoring and deliberate perceptual-motor control. In other words, accurate performance in the prism exposure situation requires allocation of limited central processing capacity to establish and maintain appropriate linkage of systems. It follows that if central processing capacity is not available, discordant systems will not be linked, performance will suffer, and adaptation will not occur. Recently, we have tested this prediction in a number of experiments employing a hall exposure procedure (Redding et al., 1985; Redding \& Wallace, 1985). Most prism-adaptation research has employed various restricted eye-hand coordination tasks. The hall exposure task is more difficult to control experimentally, but it has greater ecological validity and it prompts the greater articulation of theory necessary to identify relevant variables. Since one of our concerns has been to explain adaptation in hall exposure, our manipulations have been limited by the need to preserve the integrity of the hall exposure task.

The general strategy has been to compare level of adaptation for differently treated groups of subjects. All subjects are required to walk about hallways viewing a laterally displaced world. Experimental subjects are also required to perform a secondary cognitive task, such as mental arithmetic, simultaneous with hall exploration. The usual tests of visual and proprioceptive adaptation and total adaptation are administered before and after hall exposure. The reliable result of this manipulation is that both visual and proprioceptive adaptation are reduced by mental arithmetic, and this interference is graded, depending upon the capacity demands of the cognitive task. The more difficult the secondary task, the less adaptation occurs relative to control groups.

The basic interference effect has been replicated many times, for both optical tilt and displacement, and under a variety of conditions that seem to exclude alternative explanations. For example, interference cannot be attributed to preemption of perceptual mechanisms by the mental imagery component of the cognitive task (e.g., Segal \& Fusella, 1970) because its magnitude is unaffected by manipulations that can be assumed to vary this component. Requiring subjects to judge the angular difference between imaged hands for analog clock time does not produce greater interference than mental arithmetic, even though the clock task is at least as difficult as mental arithmetic and involves a larger mental imagery component.

Surprisingly, the primary task of walking does not suffer interference from the secondary mental arithmetic task. In a variety of conditions, walking rate has been shown to be unrelated to either performance on the secondary task or to the level of adaptation attained. To the extent that walking rate is an adequate index, interference cannot be attributed to any general decrease in exploratory locomotion and, indeed, locomotion and prism adaptation appear to be independent processes.

Figure 2 illustrates the directionality-of-guidance model for hall exposure. (The internal detail shown in Figure 1 is assumed but not shown for each sensorimotor system in Figure 2.) Visual guidance (eye-head system) of locomotion (foot-head system) is assumed not to be affected 


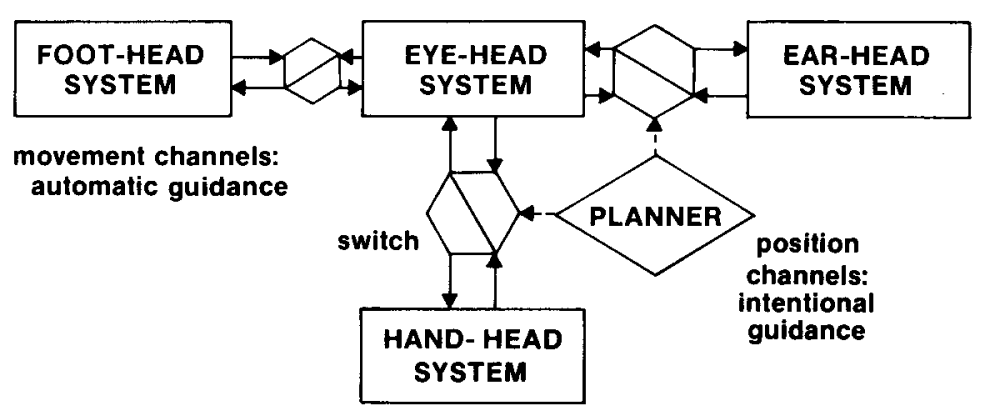

\begin{abstract}
Figure 2. Directionality-of-guidance model for hall exposure. Locomotion is automatically guided by undistorted optical flow while intentional guidance of locational responses based on distorted position is subject to interference from a secondary cognitive task with consequentially less adaptation. Directional linkages are shown in such a manner as would produce adaptation in the eye-head (visual) system. (From Redding, Clark, \& Wallace, 1985.)
\end{abstract}

by the prisms. Locomotion is guided via movement channels by Gibson-like optical flow patterns (e.g., Fitch, Tuller, \& Turvey, 1982; Lee \& Thomson, 1982) which may suffer little or no distortion by the displacing prisms. Thus, walking is assumed to be guided in the normal automatic manner, does not require central processing capacity, and does not suffer interference from the secondary cognitive task. However, the continuous nature of optical flow is such that accurate locomotion can be maintained with occasional visual response to auditory and/or proprioceptive stimuli (cf. Thomson, 1983). That is, subjects can occasionally direct their eyes to look at, for example, another person speaking (i.e., the ear-eye linkage) or an obstacle they have just bumped into (i.e., the hand-eye linkage). On these occasions, discordance and adaptive recalibration occur in the responding visual system. The direction of linkage between systems can also be reversed, with consequent change in the locus of adaptation. For instance, when a subject anticipates a proprioceptive encounter with a previously seen obstacle, discordance and recalibration occur in a proprioceptive system (e.g., hand-head). Such directional linkage of visual and nonvisual systems requires central processing capacity. When capacity is not available because it has been allocated, for example, to mental arithmetic, intersystem coordination is weakened and adaptation of any kind is reduced.

\section{Coordinative Linkage in Hall Exposure}

Thus, the model identifies two determinants of adaptation: (1) the availability of central-processing capacity to establish and maintain coordinative linkage between discordant sensorimotor systems-to the extent that such capacity is not available, the total adaptive response will be reduced in magnitude-and (2) task structure which specifies the kind and direction of coordinative linkages. The particular task demands determine the locus or loci of adaptive response. To date, our research has focused on tests of the first prediction. In this paper, we report some attempts to test the second prediction.

\section{EXPERIMENT 1}

The first experiment varied hall exposure conditions to investigate the roles of locomotion and visible sound sources in adaptation. Specifically, we wished to know first if locomotion is sufficient for adaptation in hall exposure. The directionality-of-guidance model (see Figure 2) predicts a negative answer to this question, since the locomotion sensorimotor system is assumed not to involve the positional information distorted by the prisms. Although previous findings (Redding et al., Redding \& Wallace, 1985) had supported the model, in that walking rate was not reliably affected by the imposition of a secondary cognitive load (although adaptation was) and was unrelated to level of adaptation, we wished to test the model more directly. Since adaptation is known to occur in conditions other than hall exposure (e.g., Craske, 1967), it would have been pointless to examine a nonlocomotion condition. An adequate test of the model required that the operation of the presumed sensorimotor system for locomotion be manipulated short of eliminating it. Thus, exposure conditions were created which stressed optical flow to the exclusion of other kinds of stimulus information. It was expected that salient optical flow should support high walking rates but should not produce substantial adaptation compared with conditions that prompted more controlled visual exploration.

The directionality-of-guidance model also predicts that visual adaptation should occur when visual exploration is guided by auditory input. In the very common everyday situation in which we first hear a voice and then turn our eyes (and frequently our heads also) to look at the person speaking, if the visual and auditory systems are not spatially cross-calibrated, adaptive recalibration should occur in the guided visual system, not in the guiding auditory system. It seems likely that this direction of linkage (rather than the reverse) should occur in hall exposure where sound sources would often be outside the reduced field of view. We have previously suggested (Redding \& Wallace, 1985) that the availability of visible sound 
sources (e.g., people) in the hall environment might be responsible for differences in the relative magnitude of visual and proprioceptive shift, and considering that such encounters are relatively infrequent in hall exposure environments, visible sound sources might be a primary determinant of visual adaptation. To test this hypothesis, our primary strategy was again to preserve the integrity of hall exposure while manipulating the availability of visible sound sources in a manner that as nearly as possible matched the more naturalistic situation.

\section{Method}

Subjects. The 60 subjects were right-handed undergraduate volunteers at Illinois State University. All had normal or corrected-tonormal (contact lenses only) vision.

Exposure conditions. All subjects wore a (monocular, right eye) 30-D $\left(17.1^{\circ}\right)$ rightward displacing prism and walked back and forth for $10 \mathrm{~min}$ in a short $(10.4 \mathrm{~m})$, but wide $(2.2 \mathrm{~m})$, hall, with an 8-m-long railing mounted along each wall at about shoulder height ( $1.3 \mathrm{~m}$ and $6.4 \mathrm{~cm}$ from the wall). At each end of the hall, a black curtain was hung which covered about two-thirds the width of the hall and reached to about knee height $(80 \mathrm{~cm})$ from the floor. The hall was carpeted and free of any obstacles, visible sound sources, or traffic except those introduced in the manipulations. A rectangular path $(1 \times 8 \mathrm{~m})$ was marked with gray tape $(5 \mathrm{~cm}$ wide $)$ on the gray carpet to facilitate recording of walking rate. Initially, four groups of 10 subjects each were tested with alternate assignment to conditions. Two more groups of 10 subjects each were subsequently tested to provide additional control conditions. All subjects were instructed not to look at their hands and feet, and none were observed to violate this prohibition.

Conditions for the first two groups were designed to test the value of optical flow for determining adaptation. The first group (B) experienced exaggerated optical flow intended to maximize any possible effects. These subjects were instructed to fixate a large " $\mathrm{X}$ " (each crossmember was $4 \times 30 \mathrm{~cm}$ ) on the curtain centered at each end of the hall (1.6 $\mathrm{m}$ from the floor) and to walk as rapidly as possible back-and-forth between these targets, down the center of the hallway, closing their eyes during each "about-face" turn. Thus, these subjects walked in a straight-line manner with visual input restricted to optical flow attendant upon the fixated target. Subjects in the second group (T) experienced conditions more normal for hall exposure, guiding themselves around the rectangular path but with the " $X$ " target at each end of the hall. These subjects were instructed to walk slowly toward the " $X$ " and to make a U-turn at each end of the hall. Thus, these subjects walked in a more guided manner, but optical flow was stressed by the presence of the targets. For a third (control) group (C), the target "Xs" were removed and the subjects guided themselves slowly around the rectangular path, making their U-turns at the end of the railing along each wall. Conditions for this group were intended to match the usual hall exposure with the exception that no visible sound sources were available. For all of these first three groups, the experimenter followed closely behind the subject, was never visible, and was silent except for necessary instructions at the beginning and end of the exposure period.

Conditions for the fourth group (A) matched those for the control group except that a visible sound source was added. The experimenter stood in the middle and near the end of the hall, and the subjects were instructed to walk slowly alongside the railing, passing between the experimenter and railing and making their turns behind the experimenter. While a subject was making a turn and the experimenter was not visible, the experimenter moved quickly to the opposite end of the hall and was in the subject's field of view when the turn was completed. During each period when the experimenter was visible, he provided a visible sound source by making encouraging/instructive remarks to the subject. These remarks were brief and as casual as possible. Two additional groups were tested to control for the obstacle value of the experimenter and for any general motivational effects of his remarks. For both of these groups, the experimenter's remarks were of the same kind and frequency and therefore provided the same motivation as they did for Group A; however, in this case he followed closely behind the subject and was never visible. To control for the obstacle value of the experimenter, for Group $\mathrm{O}$ chairs were placed in the positions occupied by the experimenter for Group A. Group M provided a control for motivational effects without obstacles; for these subjects, the speaking experimenter was never visible. In other respects, Groups $O$ and $M$ were similar to Group $A$.

Tests. Before and after the 10-min hall exposure, subjects were tested 10 times on each of three kinds of tests with the prism set to $0 \mathrm{D}$. The test for visual adaptation or shift (VS) required subjects to indicate verbally when a vertical line $(.2 \mathrm{~cm}$ wide and extending across the subject's visual field at a viewing distance of $60 \mathrm{~cm}$ ) appeared to be straight ahead of the nose when it was moved laterally across the visual field by the experimenter. Starting position of the test line was varied randomly $\pm 3^{\circ}$ around $15^{\circ}$ left or right from objective straight ahead, but was equally frequent in left and right visual fields. The test for proprioceptive adaptation or shift (PS) required a subject to point straight ahead of his or her nose with the right hand, with eyes closed. A total adaptation or shift (TS) measure of both VS and PS was obtained by having the subject point at the objectively straight-ahead test line with his or her unseen right hand. For both VS and TS tests, the visible field was illuminated but homogeneous except for the target line. The 30 pretests and 30 posttests were conducted in five randomized blocks of six tests: two PS, two TS, and two VS (one starting in the left visual field and the other in the right). Level of adaptation was measured by the difference in degrees between average pretest and posttest performance, the adaptive direction of change being in the direction of the displacement for the VS test and opposite the displacement for the PS and TS tests.

\section{Results and Discussion}

Table 1 lists the salient conditions and shows the primary results for each group. Separate analyses of variance were performed on the VS, PS, and walking rate

\section{Table 1}

Level of Visual Shift (VS) and Proprioceptive Shift (PS) (in Degrees) and Walking Rate (WR) (in meters/minute) for Different Conditions of Hall Exposure in Experiment 1

\begin{tabular}{|c|c|c|c|c|c|c|c|c|}
\hline Group & Target & Walking & Speed & Obstacle & Speech & VS & PS & WR \\
\hline B & $\mathrm{X}$ & B & $\mathrm{F}$ & - & - & $\begin{array}{l}-.9 \\
(1.7)\end{array}$ & $\begin{array}{c}-.4 \\
(2.0)\end{array}$ & $\begin{array}{c}62.8 \\
(5.9)\end{array}$ \\
\hline $\mathrm{T}$ & $\mathrm{X}$ & $\mathrm{G}$ & $\mathrm{S}$ & - & - & $\begin{array}{l}-.7 \\
(1.7)\end{array}$ & $\begin{array}{r}.2 \\
(2.3)\end{array}$ & $\begin{array}{c}41.4 \\
(4.7)\end{array}$ \\
\hline $\mathrm{C}$ & - & G & $\mathbf{S}$ & - & - & $\begin{array}{c}.1 \\
(1.7)\end{array}$ & $\begin{array}{c}1.7 \\
(1.9)\end{array}$ & $\begin{array}{c}40.6 \\
(4.0)\end{array}$ \\
\hline A & - & G & $\mathbf{S}$ & E & $\mathbf{Y}$ & $\begin{array}{c}2.8 \\
(1.1)\end{array}$ & $\begin{array}{l}-.4 \\
(2.4)\end{array}$ & $\begin{array}{c}41.3 \\
(6.0)\end{array}$ \\
\hline $\mathbf{M}$ & - & G & $S$ & - & $\mathrm{Y}$ & $\begin{array}{c}1.5 \\
(1.4)\end{array}$ & $\begin{array}{c}-.0 \\
(1.9)\end{array}$ & $\begin{array}{c}35.7 \\
(3.8)\end{array}$ \\
\hline $\mathrm{O}$ & - & $G$ & $S$ & C & $Y$ & $\begin{array}{c}.8 \\
(1.1)\end{array}$ & $\begin{array}{c}1.5 \\
(2.3)\end{array}$ & $\begin{array}{c}35.7 \\
(4.0)\end{array}$ \\
\hline
\end{tabular}

Note - The $95 \%$ confidence limits are shown in parentheses. The various groups were provided a target $(\boldsymbol{X})$ for walking, or there was no specific target. Subjects walked a "ballistic" straight-line path with eyes closed during turns $(B)$ or guided themselves around a rectangular path with eyes open during turns $(G)$. Subjects received instructions to walk slowly $(S)$ or fast $(F)$. Subjects experienced the experimenter $(E)$ or a chair $(C)$ as an obstacle or had no obstacle in the hall, and the speaking experimenter served as a sound source $(Y)$ or there was no systematic sound source. Thus, only Condition A had a visible sound source. 
(WR) data. For the first four groups, reliable VS (95\% confidence limits) occurred only for Group A. There were no significant differences in VS among the $\mathrm{T}, \mathrm{B}$, and $\mathrm{C}$ groups $[F(2,27)=.49]$, and VS was significantly greater for Group A than for Group C $[F(1,18)=9.53, p=$ $.006]$. Thus, there is no evidence that optical flow promotes visual adaptation, and the fact that subjects were able to walk exceedingly rapidly when instructed to do so (Group B) suggests that optical flow is available with, and not seriously distorted by, the prisms. Walking rate was reliably greater for Group B than for Group T $[F(1,18)=42.42, p<.001]$, but did not vary significantly among the five groups instructed to walk slowly $[\mathrm{F}(4,45)=2.17, \mathrm{p}=.09]$.

The visual adaptation for Group A cannot be attributed simply to the obstacle value of the visible experimenter, since Group $\mathrm{O}$ showed significantly less and unreliable VS $[F(1,18)=8.69, p=.008]$. Group $M$ produced a reliable, but intermediate, amount of VS, not significantly less than that produced by Group A $[F(1,18)=2.91$, $\mathrm{p}=.10]$, but also not significantly greater than that produced by Control Group $\mathrm{C}[\mathrm{F}(1,18)=2.21, \mathrm{p}=.15]$. Thus, the experimenter's speech may generally encourage visual locational responses, but in addition a visible experimenter provides a specific source of nonvisual guidance of visual responses with consequent discordance and adaptation in the guided visual system.

No reliable PS was obtained for any of the six groups, and no significant differences in PS appeared among the groups $[F(5,54)=.96]$. This result is not surprising, since these conditions were not designed to produce the kind of proprioceptive anticipation of visual and/or auditory targets that the model presumes necessary for PS. However, it is interesting to note that PS tended to be greatest in those conditions in which VS was small, that is, for Groups $C$ and $O$. This suggests that when there is no specific guiding source for visual responses, subjects tend to make proprioceptive locational responses. For example, they may proprioceptively anticipate encounters with the previously seen wall. Consequently, discordance and adaptation tend to be localized in proprioceptive systems.

Finally, it should be noted that the average TS (1.6) was not significantly different from the average sum $\mathrm{VS}+\mathrm{PS}(1.0)[\mathrm{F}(1,54)=2.89, \mathrm{p}=.09]$ and that there were no significant differences between these two measures for any of the groups $[F(5,54)=1.55, \mathrm{p}=.19]$. Thus, there is no basis for rejecting the assumption of additivity in these data.

\section{EXPERIMENT 2}

Locomotion per se appears to be neither necessary nor sufficient for adaptation in hall exposure. Adaptation has long been known to occur in restrictive, nonlocomotive hand exposure, and Experiment 1 demonstrated that even high walking rates and salient optical flow do not produce detectable adaptation. Consistent with the directionality- of-guidance model, locomotion does not appear to involve the sensorimotor systems directly responsible for adaptation. Adaptation does seem to depend upon task structure that requires coordinative linkage of sensorimotor systems involving distorted positional information, and auditory-visual linkage appears to be especially important for visual adaptation during free locomotion.

Experiment 2 was designed to provide a more direct test of the hypothesis that the absence of visible sound sources was responsible for the reversal in relative magnitude of VS and PS found by Redding and Wallace (1985). Usually, VS is greater than PS in hall exposure, but relative magnitude was reversed in hallways lacking the usual, noisy traffic. This second experiment was conducted in the same auditorily deprived environment as the previous study, but the availability of auditory guidance of the visual system was manipulated by making the speaking experimenter visible or not visible to the subject. The relative magnitude of VS and PS was expected to depend upon this manipulation of the experimenter.

In addition, the design of Experiment 2 provided a test of the directionality-of-guidance model against the simpler notion of "attentional weighting." Canon (1970) and Kelso et al. (1975) suggested that for two discordant modalities, adaptation occurs in the unattended modality. To the extent that this usage is different from that of the present model (see also, Redding et al., 1985), "attention" seems to refer to the relative "weight" or salience given to a stimulus source in phenomenal experience (consciousness). This directed-attention hypothesis might explain cognitive interference in the following manner. Arithmetic problems given auditorily by a nonvisible experimenter direct attention to the auditory modality and away from other information sources (e.g., visual and proprioceptive). Since the sound source is not visible, it is not discordant with vision and, thus, arithmetic problems reduce adaptation by reducing experienced discordance. Discordant modalities (e.g., vision and proprioception) are equally unweighted and the stimulus for adaptation is reduced. If this is the case, then arithmetic problems given by a visible experimenter should actually facilitate adaptation relative to a condition in which the experimenter is visible but largely silent. For instance, with a visible speaking experimenter, vision and audition are discordant and the problems should direct attention to the auditory modality, thereby prompting adaptation in the unattended visual modality. In contrast, the directionality-of-guidance hypothesis makes the opposite prediction. A visible speaking experimenter should produce more visual adaptation than would conditions in which the experimenter was not visible because any auditory stimuli from the visible sound source should serve as guidance signals for visual locational responses, but all else being equal arithmetic problems should usurp processing capacity and reduce the frequency of coordinative linkage between discordant systems.

The method employed was the same as in Redding and Wallace (1985), except that only the most difficult set of 
arithmetic problems was used and the four groups received problems or no problems with the experimenter visible or not visible. The experimenter either followed closely behind the subject or stood at the end of the hall, clearly visible to the subject. In the no-problem conditions, conversation with the subject was minimized. The directionality-of-guidance model predicts independent effects of cognitive load (problems or no problems) and task structure (experimenter visible or not) on level and locus of adaptation, respectively. Interference should occur as in previous studies, with both visual and proprioceptive adaptation being reduced when arithmetic problems were given. However, within each level of the problems variable, the relative magnitude of visual and proprioceptive adaptation should depend upon whether or not the experimenter was visible, providing an auditory stimulus for visual locational responses.

\begin{abstract}
Method
Subjects. The 60 subjects were right-handed undergraduate volunteers at Cleveland State University. All had normal or correctedto-normal (contact lenses only) vision.

Exposure conditions. All subjects walked back and forth along a short hall $(9.1 \mathrm{~m}$ in length $\times 1.7 \mathrm{~m}$ in width) for $10 \mathrm{~min}$, wearing goggles which afforded a monocular (right eye) view through a Risley prism set to laterally displace the visual field $30 \mathrm{D}\left(17.1^{\circ}\right)$ in the rightward direction. Each subject walked a path down one side of the hall, made a U-turn near the end, and walked back down the other side. Pieces of tape (not visible to the subject) on the floor facilitated recording of walking rate. Four groups of 15 subjects each received different treatments determined by the factorial combinations of problems or no problems and experimenter visible or not.
\end{abstract}

The two problem groups received arithmetic problems created by generating all the unordered pairs of the double-digit numbers from 70 to 99 , excluding identical pairs. These 435 problems were ordered randomly prior to the experiment and were presented aloud in the form of addition questions (e.g., "'95+79=?'). The subjects were required to solve as many problems as they could as accurately and as quickly as possible while walking in the hall, and the accompanying experimenter recorded the number of problems attempted and the number correctly solved. For the two groups not receiving problems, verbal interaction with the experimenter was minimized. Although exchanges could not be eliminated entirely, an effort was made to restrict the experimenter's contribution to those times when she was not visible to the subject.

For the two conditions in which the experimenter was visible to the subject, the experimenter stood in the middle and toward the end of the hall, and the subject made his or her turn behind the position of the experimenter. While a subject was making a turn and the experimenter was not visible, the experimenter moved quickly to the opposite end of the hall and was present in the subject's field of view when the turn was completed. For the two conditions when the experimenter was not visible, the experimenter followed a path directly behind the subject and never appeared in the subject's visual field.

The hall was the same evacuated one used by Redding and Wallace (1985), and there was little human traffic except for the experimenter and subject. However, a restroom located in the hall was the occasion for infrequent pedestrian incursions from other parts of the building, and maintenance personnel also occasionally appeared in the hall. Although speech did not usually accompany such stimuli, there were associated sounds of footsteps and doors. The only other detectable source of auditory stimuli was a nonvisi- ble elevator. The hall was also free of any obstacles and was interrupted only by the facings of closed doors.

As in previous experiments (Redding et al., 1985; Redding \& Wallace, 1985), subjects not receiving problems were instructed to walk slowly and those receiving problems were encouraged to walk rapidly. The latter instruction was intended to counter the natural tendency for problem solving to slow walking rate, and to control for the possibility that reduced adaptation might be attributable to a general reduction in exploratory behavior. ${ }^{3}$ Finally, as in the previous experiment, all subjects were instructed not to look at their hands or feet and none were observed to violate this prohibition.

Tests. The tests were similar to those used in Experiment 1, with the following exceptions. The test line for VS and TS tests was only $8 \mathrm{~cm}$ long and was vertically centered in the subject's field of view. For PS tests, the subject's eyes were open to a monocular (right eyes) and homogeneous white field. All 10 measures of one kind of test were obtained before another kind of test was given, but the order of tests was otherwise random across subjects. Finally, starting position for the VS tests was random but equally frequent in left and right visual fields. (For additional details of test procedures and apparatus, see Wallace \& Redding, 1979.)

\section{Results and Discussion}

Directional linkage. A mixed-design analysis of variance was performed on VS and PS data for subjects in the four independent groups. The results of primary interest are displayed in Figure 3. The average level of VS $\left(2.4^{\circ}\right)$ and PS $\left(2.7^{\circ}\right)$ was not statistically different $[F(1,56)=1.18, p=.28]$, but VS was reliably greater than PS when the experimenter was visible and PS was reliably greater than VS when the experimenter was not visible $[\mathrm{F}(1,56)=24.16, \mathrm{p}<.001]$, and this pattern was not statistically different for problem and no-problem conditions $[\mathrm{F}(1,56)=1.09, \mathrm{p}=.30]$. Thus, it appears that even infrequent auditory stimuli from the visible experimenter are sufficient to initiate visual locational responses with consequent discordance and recalibration in the visual system. When the experimenter is not visible, there are fewer occasions for such visual response, the subject responds proprioceptively to visual targets (proprioceptive anticipation), with consequent discordance and recalibration in the proprioceptive system. ${ }^{4}$

It should also be noted that differences in locus of adaptation cannot be attributed to differential "attention" to the secondary task. The percentage of correctly solved problems was the same whether the experimenter was visible $(85)$ or not $(86)[F(1,28)<1.00]$, and neither were there any significant differences between these groups in terms of the number of problems attempted $[F(1,28)=$ $1.82]$ or the total number of correctly solved problems $[\mathrm{F}(1,28)=1.28]$. Walking rate (meters/minute) was also the same whether the experimenter was visible $(60.8)$ or not $(60.3)[F(1,56)<1.00]$, and there was no interaction of the problems and experimenter variables for this dependent measure $[F(1,56)=1.92, p=.17]$.

Interference. As in previous studies (Redding, Clark, \& Wallace, 1985; Redding \& Wallace, 1985), arithmetic problems interfered with adaptation $[F(1,56)=7.00, p$ $=.01 \mathrm{~J}$. Also like previous studies, interference cannot 


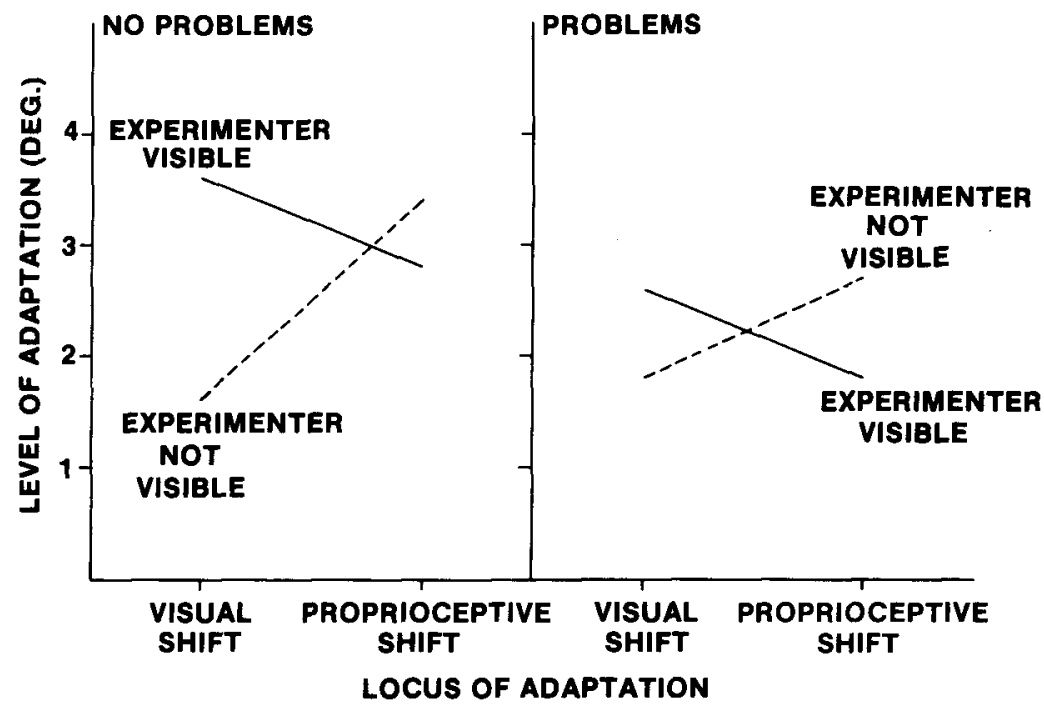

Figure 3. Level of visual and proprioceptive shift as a function of whether or not arithmetic problems were given and whether or not the experimenter was visible during hallway walking (Experiment 2).

be attributed to any decrease in general exploration, since the problems groups actually walked faster $(69.4 \mathrm{~m} / \mathrm{min})$ than groups not receiving problems $(51.6 \mathrm{~m} / \mathrm{min})[\mathrm{F}(1,56)$ $=104.14, \mathrm{p}<.001]$. The magnitude of interference was essentially the same for both kinds of adaptation $[F(1,56)$ $=1.12, \mathrm{p}=.29 \mathrm{~J}$, but there was a tendency toward greater interference when the experimenter was visible $[\mathrm{F}(1,56)$ $=2.84, p=.09$ ], perhaps because the higher overall level of adaptation makes this condition more sensitive to interference. Nevertheless, the general pattern suggests that the problems and experimenter variables are independent factors, consistent with the idea that coordinative linkage and the direction of such linkage are separate processes, affected by the availability of central processing capacity and task structure, respectively.

Clearly, there is no evidence of any simple attentional weighting. Interference occurred for both kinds of adaptation and for all groups, even when the experimenter was visible and auditory "attention" should have been expected to result in facilitation of adaptation in "unattended" visual and/or proprioceptive modalities. Attentional involvement in adaptation seems to be more than the simple weighting of an information source in conscious awareness. These results are at least consistent with the notion of limited attentional capacity to establish coordinative linkages between discordant systems.

Additivity. The average TS (5.4) was slightly, but significantly, greater than the average of the sum VS + PS $(5.1)[F(1,56)=4.22, p=.04]$, although this difference was restricted entirely to the group not receiving problems and with the experimenter not visible $[\mathrm{F}(1,56)=8.25$, $\mathrm{p}=.005]$. For this group, TS (6.2) was $1.2^{\circ}$ greater than the sum VS + PS (5.0). The reason for this "underadditivity" is not known, but it should be noted that it occurred under conditions in which the task structure af- forded the least coordinative linking of discordant systems. Under such conditions, any purely local bias would remain uncorrected, since there is little occasion for the two discordant systems to be brought into cross-calibration with each other. Thus, although the exact reason for the nonadditivity is problematic at present, it is at least consistent with the model in the sense that it occurs under those conditions in which cross-calibration is least likely.

\section{EXPERIMENT 3}

Experiment 2 suggests that visible sound sources are a powerful determinant of visual adaptation. In terms of the directionality-of-guidance model, this variable is assumed to affect the direction of coordinative linkage between discordant systems independent of cognitive load, which is assumed to affect the degree of intersystem linkage, regardless of direction. Thus, the model predicts an additive relationship between the problems and experimenter factors. The tendency toward interaction found in Experiment 2 might have been due to a failure to equate the problems conditions in terms of visible sound sources. Even infrequent visible sound sources may have large effects. Experiment 3 attempted to achieve better control over visible sound sources by using a minimally difficult problem set in place of the no-problems condition. Thus, all subjects experienced sound sources but differed in cognitive load and whether the sound source was visible or not.

\section{Method}

The method was the same as in Experiment 2, except that only 12 subjects were tested in each of the four groups and the noproblems conditions were replaced by the minimally difficult problem set used by Redding and Wallace (1985). This set con- 
sisted of the 36 single-digit probiems created by generating all the unordered pairs of the single digits from 1 to 9 , excluding identical pairs, randomly ordered in repeated blocks prior to the experiment and presented aloud to subjects in the form of addition questions (e.g., "3 $3=4=$ ?"). Redding and Wallace (1985) demonstrated that this problem set is less difficult and produces less interference with adaptation than do the double-digit problems.

\section{Results and Discussion}

The primary results are illustrated in Figure 4. Doubledigit problems produced less adaptation of either kind than did single-digit problems $[\mathrm{F}(1,44)=9.79, \mathrm{p}=.003]$, and, as in Experiment 2, the relative magnitude of VS and PS depended upon whether the experimenter was visible or not $[F(1,44)=10.49, p=.002]$. No other source of variance was significant. Thus, as predicted by the model, cognitive load and task structure appear to be independent determinants of magnitude and locus of adaptation, respectively.

Unlike the previous experiment, these data show perfect additivity. The average TS and the average sum VS + PS were identical for all four groups, and the problems variable was the only significant source of variance in these analyses. Thus, additivity occurs when conditions are such as to promote cross-calibration of sensorimotor systems and total adaptation is not affected by those aspects of task structure which simply determine the direction of coordinative linkage between systems.

The relative difficulty of the two problem sets was reflected in all measures of performance. For example, the average percentage of correctly solved problems was greater for single-digit problems (99) than for double-digit problems $(76)[F(1,44)=216.67, p<.001]$. There were 11.5 fewer problems attempted when the experimenter was visible than when she was not visible $[\mathrm{F}(1,44)=$ $5.67, \mathrm{p}=.02$ ] and 10.2 fewer problems solved correctly when the experimenter was visible than when she was not visible $[F(1,44)=5.06, p=.03]$, but the percentage of correctly solved problems was not significantly different whether the experimenter was visible (87) or not (88) $[F(1,44)<1.00]$, and there were no significant interactions for these dependent measures. Walking rate was the same whether the experimenter was visible $(59.2 \mathrm{~m} / \mathrm{min})$ or not $(58.5 \mathrm{~m} / \mathrm{min})[\mathrm{F}(1,44)<1.00]$, and people receiving the double-digit problems walked faster $(65.0 \mathrm{~m} / \mathrm{min})$ than those receiving single-digit problems $(52.7) \mathrm{m} / \mathrm{min}$ ) $[F(1,44)=56.30, p<.001]$, regardless of level of the experimenter factor $[F(1,44)<1.00]$.

\section{CONCLUSIONS}

Clearly, locomotion per se appears to be neither necessary nor sufficient for prism adaptation in hall exposure. Walking rate is not reliably related to level of adaptation and does not suffer interference in the way that adaptation is affected by a secondary cognitive load (Redding et al., 1985; Redding \& Wallace, 1985). Furthermore, when the hall exposure task is restricted to a locomotion task, no detectable adaptation occurs. We believe that the available data strongly support a model that places locomotion under the control of a sensorimotor system that is not involved in adaptation to optical displacement. We further suggest that the prismatic distortion may not affect optical flow and the subject can locomote in the normal, unmonitored manner. However, the precise effect of displacing prisms on optical flow is problematical, since the nature of the computational mechanisms that extract information from flow patterns is not completely understood (but see Longuet-Higgins \& Prazdny, 1980). Perhaps the strongest conclusion possible at the present time is that locomotion and prism adaptation are mediated by separate mechanisms in hall exposure.

The presence of a coordinative linkage between discordant sensorimotor systems seems to be a primary determinant of adaptation, and when the use of nonhabitual

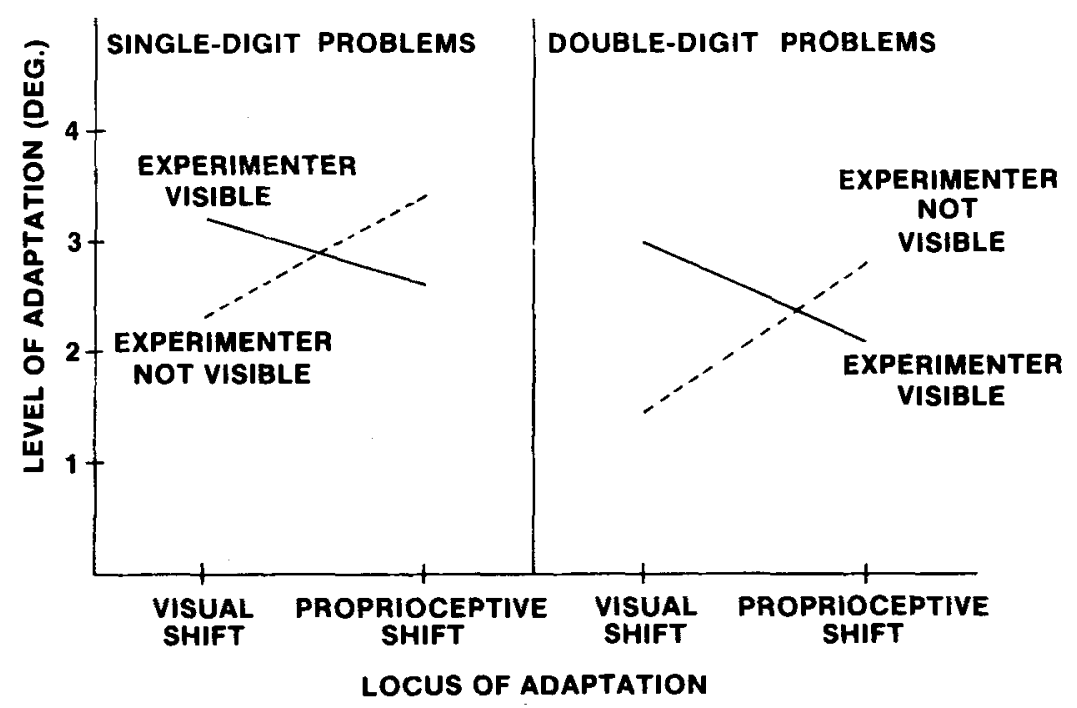

Figure 4. Level of visual and proprioceptive shift as a function of the difficulty of problems given and whether or not the experimenter was visible during hallway walking (Experiment 3). 
linkages required for coordination during prism exposure is reduced by the imposition of a secondary cognitive load, total adaptation is reduced. The distribution of total adaptation among the several discordant systems may be determined by the direction of coordinative linkages afforded by task structure. An important aspect of task structure in hall exposure appears to be the presence of visible sound sources, which may be presumed to serve as a source of guidance signals for visual locational responses with consequent discordance and adaptation in the guided visual system. In the absence of visible sound sources, subjects may use visual information to guide proprioceptive anticipation of object location (as when the hand is extended prior to touching the previously seen wall) with resultant local discordance and adaptation in proprioceptive systems. $^{5}$

Our attempts to understand adaptation in hall exposure have proven highly heuristic, but although we regard continued efforts in this direction as necessary for generalization of the model beyond the laboratory, we also recognize the difficulties in doing research in more naturalistic settings. Testing fine details of the model requires the greater experimental control afforded by more restricted situations. We are currently attempting to determine those factors with hand exposure which might determine direction of guidance. For instance, specifying a proprioceptive target for a subsequent visual locational response may establish a hand-to-eye direction of linkage with resultant visual adaptation.

\section{REFERENCES}

CANON, L. K. (1970). Intermodality inconsistency of input and directed attention as determinants of the nature of adaptation. Journal of Experimental Pscyhology, 84, 141-147.

Craske, B. (1967). Adaptation to prisms: Change in internally registered eye-position. British Journal of Pscyhology, 58, 329-335.

Craske, B., \& CrawshaW, M. (1974). Adaptive changes of opposite sign in the oculomotor systems of the two eyes. Quarterly Journal of Experimental Psychology, 26, 106-113.

Craske, B., \& Crawshaw, M. (1978), Spatial discordance is a sufficient condition for oculomotor adaptation to prisms: Eye muscle potentiation need not be a factor Perception \& Psychophysics, 23, 75-79.

CRAWSHAW, M., \& CRASKE, B. (1974). No retinal component in prism adaptation. Acta Psychologica, 38, 421-423.

EBENHOLTZ, S. M. (1976). Additivity of aftereffects of maintained head and eye rotations: An alternative to recalibration. Perception \& Psychophysics, 76, 113-116.

FISHER, G. H. (1962a). Intersensory localization. Unpublished doctoral thesis, University of Hull, England.

FisHeR, G. H. (1962b). Resolution of spatial conflict. Bulletin of the British Pscyhological Society, 46, 3A.

Fitch, H. L., Tuller, B., \& Turvey, M. T. (1982). The Bernstein perspective: III. Tuning of coordinative structures with special reference to perception. In J. A. S. Kelso (Ed.), Human motor behavior. Hillsdale, NJ: Erlbaum.

HamiLton, C. R. (1964). Studies on adaptation to deflection of the visual field in split-brain monkeys and man. Unpublished doctoral dissertation, California Institute of Technology.

Harris, C. S. (1965). Perceptual adaptation to inverted, reversed, and displaced vision. Psychological Review, 72, 419-444.

HaRRIS, C. S. (1980). Insight or out of sight? Two examples of percep- tual plasticity in the human adult. In C.S. Harris (Ed.), Visual coding and adaptability. Hillsdale, NJ: Erlbaum.

Held, R., \& Bossom, J. (1961). Neonatal deprivation and adult rearrangement: Complementary techniques for analyzing plastic sensorymotor coordination. Journal of Comparative \& Physiological Psychology, 54, 33-37.

Howard, I. P. (1971). Perceptual learning and adaptation. British Medical Bulletin, 27, 248-252.

HowARD, I. P. (1982). Human visual orientation. New York: Wiley. HowARD, I. P., \& Templeton, W. B. (1966). Human spatial orientation. New York: Wiley.

KALIL, R. E., \& FREEDMAN, S. J. (1966). Intermanual transfer of compensation for displaced vision. Percepnual \& Motor Skills, 22, 123-126.

Kelso, J. A. S., Cook, E., Olson, M. E., \& EPSTein, W. (1975). Allocation of attention and the locus of adaptation to displaced vision. Journal of Experimental Psychology: Human Perception and Performance, 1, 237-245.

LeE, D. N., \& Thomson, J. A. (1982). Vision in action: The control of locomotion. In D. J. Ingle, M. A. Goodale, \& R. J. W. Mansfield (Eds.), Analysis of visual behavior. Cambridge, MA: MIT Press.

Longuet-Higgins, H. C., \& Prazdny, K. (1980). The interpretation of a moving retinal image. Proceedings of the Royal Society of London, Series B, 208, 385-397.

POSNER, M. I., \& SNYDER, C. R. R. (1975). Attention and cognitive control. In R. L. Solso (Ed.), Information processing and cognition. Hillsdale, NJ: Erlbaum.

Redding, G. M. (1978). Additivity in adaptation to optical tilt. Journal of Experimental Psychology: Human Perception and Performance, 4, $178-190$.

Redding, G. M., Clark, S. E., \& Wallace, B. (1985). Attention and prism adaptation. Cognitive Psychology, 17, 1-25.

Redding, G. M., \& Wallace, B. (1976). Components of displacement adaptation in acquisition and decay as a function of hand and hall exposure. Perception \& Psychophysics, 20, 453-459.

REDDING, G. M., \& WALLACE, B. (1978). Sources of "overadditivity" in prism adaptation. Perception \& Psychophysics, 24, 58-62.

Redding, G. M., \& Wallace, B. (1985). Cognitive interference in prism adaptation. Perception \& Psychophysics, 37, 225-230.

RoBINSON, D. A. (1976). Adaptive gain control of vestibuloocular reflex by the cerebellum. Journal of Neurophysiology, 39, 954-969.

SChNeIder, W., \& SHIFFrin, R. M. (1977). Automatic and controlled information processing in vision. In D. LaBerge \& S. J. Samuels (Eds.), Basic processes in reading. Hillsdale, NJ: Erlbaum.

SEGAL, S. J., \& FuSELla, V. (1970). Influence of imagining pictures and sounds on detection of visual and auditory signals. Journal of Experimental Psychology, 83, 458-464.

Templeton, W. B., Howard, I. P., \& Wilkinson, D. A. (1974). Additivity of components of prismatic adaptation. Perception \& Psychophysics, 15, 249-257.

THOMSON, J. A. (1983). Is continuous visual monitoring necessary in visually guided locomotion? Journal of Experimental Psychology: Human Perception and Performance, 9, 427-443.

UHLARIK, J. J., \& CANON, L. K. (1971). Influence of concurrent and terminal exposure conditions on the nature of perceptual adaptation. Journal of Experimental Psychology, 9, 233-239.

WALLACE, B. (1977). Stability of Wilkinson's linear model of prism adaptation over time for various targets. Perception, 6, 145-151.

WALLACE, B., \& REDDING, G. M. (1979). Additivity in prism adaptation as manifested in intermanual and interocular transfer. Perception \& Psychophysics, 25, 133-136.

WELCH, R. B. (1974). Speculations on a model of prism adaptation. Perception, 3, 451-460.

Welch, R. B. (1978). Perceptual modification. New York: Academic Press.

Welch, R. B., Choe, C. S., \& Heinrich, D. R. (1974). Evidence for a three-component model of prism adaptation. Journal of Experimental Psychology, 103, 700-705.

WILKINSON, D. A. (1971). Visual-motor control loop: A linear system? Journal of Experimental Psychology, 89, 250-257. 


\section{NOTES}

1. The term "visual adaptation" is used to designate adaptive change in the eye-head system that has phenomenal consequences for visual perception. The basic nature of such adaptation may be a change in either retinal local sign or registered eye position (e.g., Crawshaw \& Craske, 1974; Harris, 1980). Current theory does not permit a test between these two possible accounts of visual change, and the present research is neutral with respect to this problem. "Proprioceptive adaptation" refers to any change in felt position of body parts. In the present study, the tests are sensitive to any such proprioceptive shift between the hand and head.

2. The term "automatic" is operationally defined as the absence of interference from the imposition of a secondary cognitive load (e.g., Posner \& Snyder, 1975; Schneider \& Shiffrin, 1977).

3. This manipulation was originally intended to equate walking rates for experimental and control groups (Redding et al., 1985), and when instruction is enforced as necessary and repeated admonitions are given during the exposure period, it produces a faster walking rate for experimental groups (Redding \& Wallace, 1985) and provides an even stronger control for any decrease in exploratory locomotion when problems are given.

4. Further evidence that it is visible sound sources, not just sound sources, that are important comes from an examination of the 19 subjects (approximately equally distributed among the four groups) who experienced the nonvisible elevator noise. Comparison of VS, PS, and TS data for the presence or absence of this nonvisible sound source revealed no significant effects involving this variable.

5. It has been suggested that the effects of experimenter visibility might have arisen from differential head and eye postures in the two conditions. Of course, eye and head postures per se are not relevant for the kind of perceptual recalibration depicted in Figure 1, but the more peripheral muscle potentiation mechanism suggested by Ebenholtz (e.g., 1976) would be sensitive simply to asymmetric postures. There does not appear to be any obvious way to directly control for such effects and still maintain the integrity of hall exposure, but there are at least two reasons we do not believe that there were substantial muscle potentiation effects in these experiments. First, asymmetric head postures were not so large as to be remarkable. Large head turning does tend to occur when the combination of distortion and hallway structure (e.g., leftward displacement and a near right wall) prevents a clear view of the hall, but the present situation did not produce noticeable head turning in any of the conditions. Second, even if there were small amounts of head turning, there does not seem to be any reasonable combination of eye and head postures which would have produced the obtained changes of opposite direction in VS and PS. For instance, PS could be increased by a leftward head position, but then the eyes would have to be turned more rightward to maintain a view of the hall and an increase in VS would then be expected.

(Manuscript received May 16, 1985;

revision accepted for publication October 7,1985 .) 